\title{
Emergency medicine residents' ability to identify ocular pathology in a live porcine model
}

\author{
Vincent L. Ball · Bradley N. Younggren • \\ Jason D. Bothwell
}

Received: 15 April 2010/Accepted: 26 January 2011/Published online: 17 February 2011

(C) Springer-Verlag (outside the USA) 2011

\begin{abstract}
Objective To evaluate emergency medicine residents' ability to detect ocular pathology using portable ultrasound (US) in a live porcine model.

Methods This was a pilot study to evaluate emergency medicine residents' ability to accurately diagnose ocular pathology in a porcine model using handheld US. Subjects were emergency medicine residents who had all undergone a 2-day US course as part of their emergency medicine orientation month and had reviewed an $1 \mathrm{~h}$ self-instruction computer tutorial on ocular US prior to the study. Vitreous hemorrhages, retro-orbital hematomas and intraocular foreign bodies were simulated by placement of porcine blood and metallic objects under ultrasound guidance out of view of the subject population. Residents then performed selfdirected US of two eyes each and were asked to comment on any pathology observed. Some residents were also asked to identify central retinal artery flow. Time required for each scan was noted.

Results A total of 36 residents scanned 6 porcine eyes over 2 lab iterations. EM residents were able to detect a significant abnormality greater than $93 \%$ of the time. Vitreous hemorrhages were the most detectable injuries with 95\% accuracy. A significant abnormality was detected in the models with intraocular foreign bodies $97 \%$ of the time with a clear diagnosis of foreign body noted in $73 \%$ of the cases. Retro-orbital hematoma was the most difficult to detect with $62 \%$ accuracy. The average time taken for scanning two eyes was 7 min $38 \mathrm{~s}$. Central retinal artery flow was detected in $100 \%$ of the 26 cases in which this
\end{abstract}

V. L. Ball $(\bowtie) \cdot$ B. N. Younggren · J. D. Bothwell

Department of Emergency Medicine, Madigan Army Medical

Center, Fort Lewis, WA 98431, USA

e-mail: docball40@gmail.com was documented. Accuracy of diagnoses was similar across levels of EM training.

Conclusion EM residents can accurately diagnose significant ocular pathology using handheld US in a live porcine model.

Keywords Ocular ultrasound - Bedside ultrasound . Porcine model

\section{Introduction}

Each year approximately 1 million patients present to an emergency department (ED) in the United States with ocular injuries [1]. The ability to rapidly diagnose ocular pathology with certainty allows the emergency physician to make disposition, transfer, or consultation decisions within minutes. The most recent American College of Emergency Physicians policy statement lists ocular ultrasound as a core application with emphasis on identifying posterior chamber abnormalities, retinal detachment, vitreous hemorrhage, and possibly elevated intracranial pressure reflected sonographically by increased optic nerve sheath diameter [2,7]. Previous cases reports and case series have utilized bedside emergency medicine ultrasound to identify intraocular foreign bodies, ruptured globes, vitreous hemorrhage, retro-bulbar hemorrhage, and retinal detachments $[3,4]$. A previous study utilized harvested porcine eyes to identify emplaced foreign bodies [6]. Central retinal artery flow can be detected via ultrasound and may be important in ruling out central retinal artery occlusion. The modality is especially useful in remote environments or when the patient is too unstable to leave the ED for imaging and in patients in whom soft tissue swelling would preclude eyelid retraction and detailed physical examination [5, 7]. 
While the potential uses of bedside ocular ultrasound are expanding, there remains a lack of data as to the sensitivity and specificity of the modality in identifying pathology at the hands of the emergency physician. This is a pilot study to evaluate emergency medicine residents' ability to detect ocular pathology in a live porcine model.

\section{Methods}

\section{Study design}

This was a single-blinded outcome study conducted at a large suburban Army Medical Center that is a level II trauma center with an annual ED census of 78,000 patients. The study population included on-service residents from an emergency medicine residency program comprised of 12 residents per year in a 3-year program. As part of quarterly training, the residents perform procedures on intubated, anesthetized, and paralyzed pigs in a co-located veterinary facility. Procedures normally performed include internal cardiac pacing, tube thoracostomy, pericardiocentesis, and thoracotomy. The study took place as an adjunct to the normal procedure lab. Each of the residents had previously undergone a 2-day ultrasound course as part of his orientation month. The week prior to the lab, each resident performed a $1 \mathrm{~h}$ power-point ocular ultrasound tutorial which illustrated proper ocular ultrasound technique and normal and pathologic ocular images from published literature. Ocular ultrasound technique has been described previously elsewhere and includes utilizing a linear probe and scanning through a closed eyelid $[3,6,8]$. None of the residents had significant previous experience in ocular ultrasound. This study was approved by the Institutional Review Board and the Institutional Animal Care and Use Committee.

The research personnel manipulated the pig eyes out of view of the ED residents and had individual residents perform ocular ultrasound on the manipulated eyes behind a screen similar to an anesthesiology drape found in the operating room. A total of three pigs with six healthy eyes were utilized over two quarterly rotations with 2 pigs utilized during iteration one and 1 pig on the second iteration. Not all residents are present at each lab due to clinical rotation scheduling. Additionally, two EM Physician Assistant fellows participated in the study and were assigned as EM-1s. The purpose of the first lab was to determine the residents' ability to identify vitreous hemorrhages and retro-bulbar hematomas and the purpose of the second iteration was to determine the residents' ability to identify intraocular foreign bodies and central retinal artery flow. Each of the six eyes contained one pathologic abnormality including vitreous hemorrhages in two eyes, retro-bulbar hematomas in two eyes, and intraocular metallic foreign bodies in two eyes. For the first iteration each of the two pigs had a vitreous hemorrhage in one eye and a retro-bulbar hematoma in the other. For the vitreous hemorrhages and retro-bulbar hematomas $10 \mathrm{ml}$ of porcine blood was placed under ultrasound guidance by a senior resident and staff emergency physician (Figs. 1, 2). For the second iteration the pig had an implanted foreign body in each eye. The foreign bodies were manipulated 14-gauge lead BBs, formed into smooth 9-mm-long cylindrical objects and introduced with a lumbar puncture stylet through a 16-gauge needle. One of the lead foreign bodies was placed in the mid-vitreous and one was placed in the posterior vitreous adjacent to the retina under ultrasound guidance (Figs. 3, 4). A Sonosite Veterinary Model, equivalent to the portable Sonosite 180 with a general purpose linear $10 \mathrm{MHz}$ transducer, with adequate gel was positioned at the head of the pig (Fig. 5). Residents were

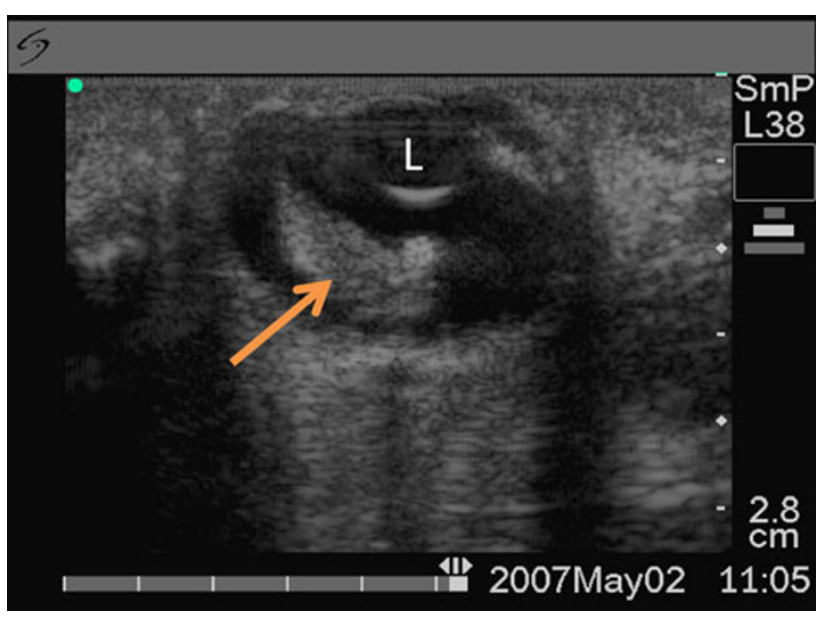

Fig. 1 Arrow indicating vitreous hemorrhage. $L$ lens

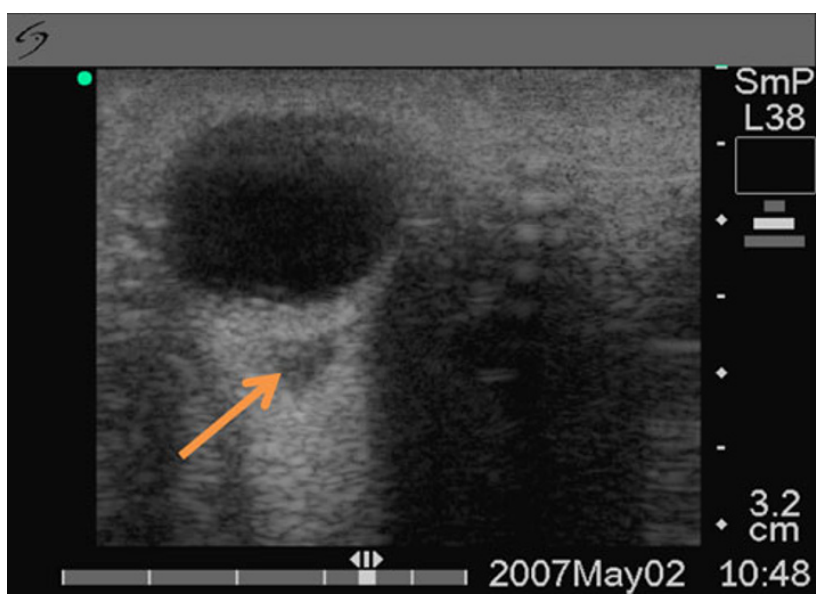

Fig. 2 Arrow indicating retro-bulbar hematoma 


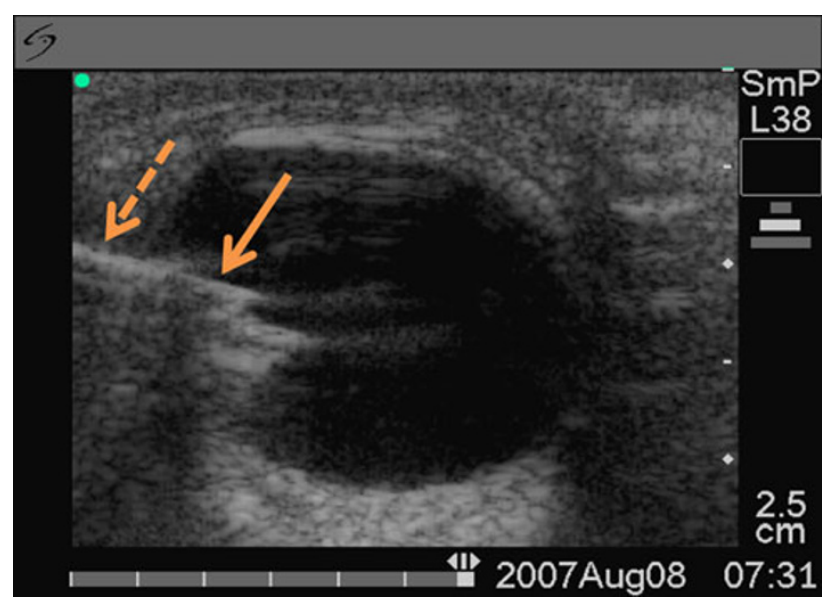

Fig. 3 Stylet (solid arrow) inserting foreign body through a needle (dashed arrow) into mid-vitreous

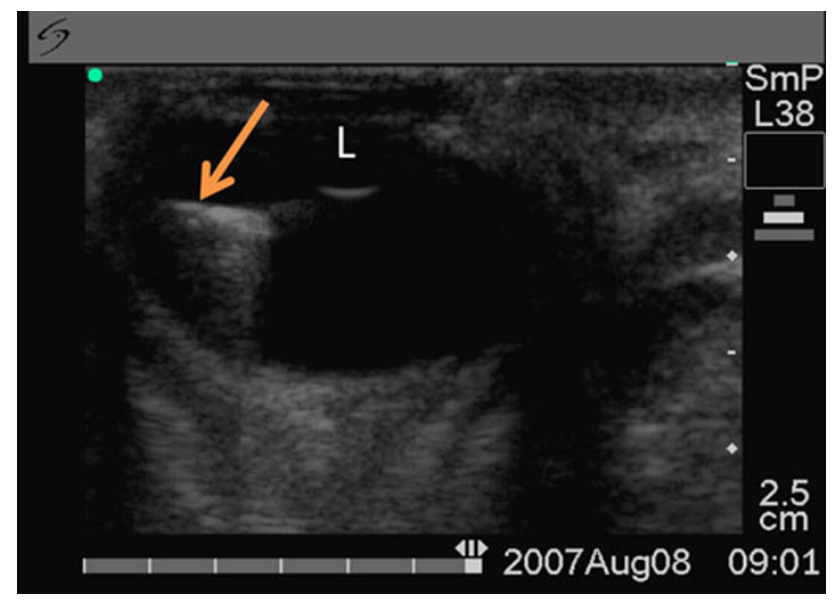

Fig. 4 Arrow indicating linear mid-vitreous foreign body after repeated scans. Note posterior reverberation. $L$ lens

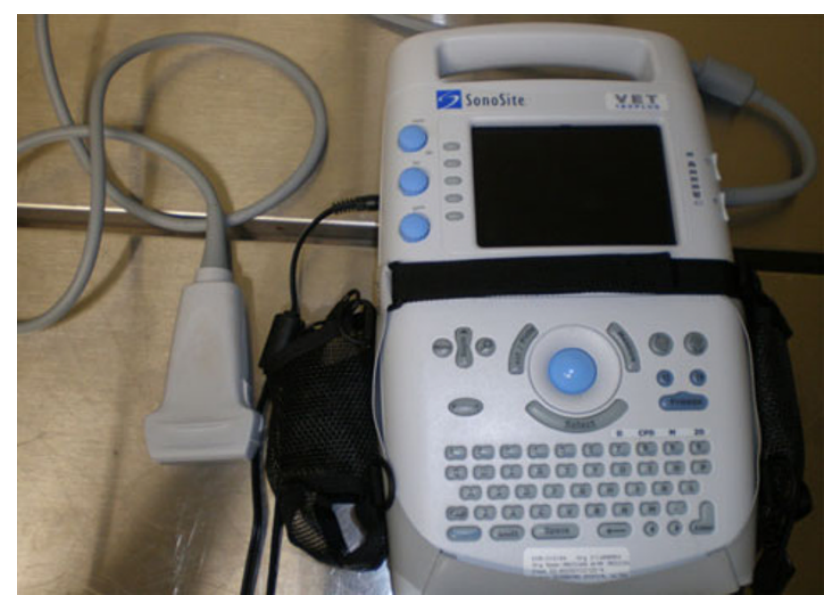

Fig. 5 Sonosite 180 with linear $10 \mathrm{mHz}$ probe

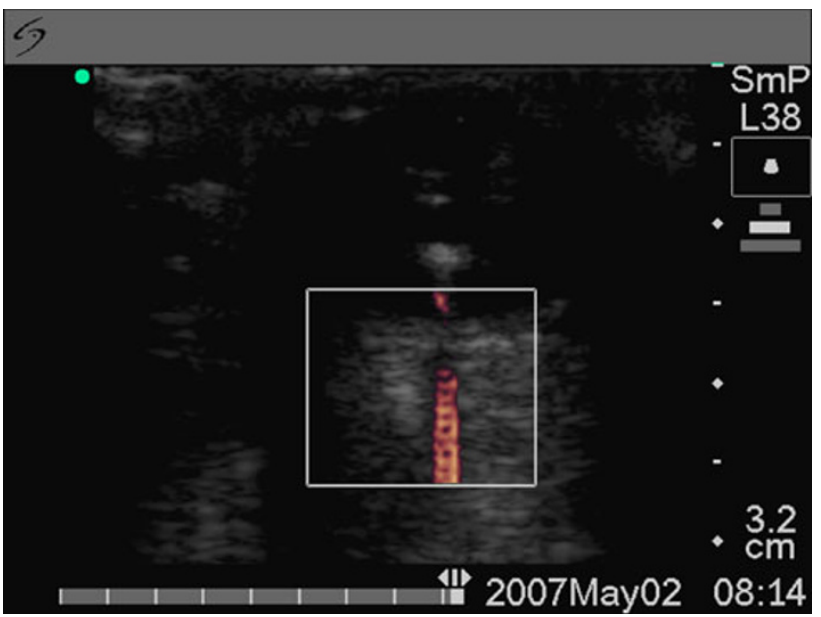

Fig. 6 Normal pulsatile central retinal artery flow

given unlimited time and were allowed to utilize all knobs, including color flow Doppler. The resident was not given clinical information but was instructed to perform an ocular ultrasound and identify and diagnose any identified pathology. Research personnel documented both the time taken for the ocular scan and the resident's final imaging diagnosis (abnormal vs. normal as well as the specific diagnosis). During the second quarterly rotation, residents were asked to identify central retinal artery flow which remained intact as the retro-bulbar area had not been disturbed as it had when vitreous and retinal hematomas were manipulated in the first quarterly rotation. Residents were asked to identify centrally located pulsatile flow (Fig. 6). Residents were not allowed to discuss their scans with other residents and the level of discussion among the 3-4 residents performing procedures on the body of the animal masked the whispered responses of the resident sonographer to the research personnel and prevented cross-contamination. All pigs were humanely euthanized under veterinary supervision at the end of the lab/study.

Data analysis

Identification of abnormality as well as accuracy of diagnosis, average scan time of both porcine eyes and identification of central retinal artery flow were calculated. Table 1 summarizes the results by all three year groups over both iterations. The residents rotated into the veterinary lab as available and not the same number scanned the pigs in each iteration.

\section{Results}

During the two procedure labs a total of 36 residents scanned a total of 6 eyes to evaluate for vitreous hemorrhage, retro- 
Table 1 Ocular scan results

\begin{tabular}{|c|c|c|c|c|c|c|c|c|c|}
\hline & \multicolumn{2}{|c|}{$\begin{array}{l}\text { VH }(2 \text { eyes, iteration } 1 \\
\text { residents scanned } 1)\end{array}$} & \multicolumn{2}{|c|}{$\begin{array}{l}\text { RH ( } 2 \text { eyes, iteration } 1 \\
\text { residents scanned } 1)\end{array}$} & \multicolumn{2}{|c|}{$\begin{array}{l}\text { IOFB ( } 2 \text { eyes, iteration } 2 \\
\text { residents scanned } 2 \text { ) }\end{array}$} & \multirow{2}{*}{$\begin{array}{l}\text { CRA flow } \\
\text { ( } 2 \text { eyes, iteration } 2) \\
\text { Present? }\end{array}$} & \multicolumn{2}{|l|}{ Scan time } \\
\hline & Abnl & Accuracy & Abnl & Accuracy & Abnl & Accuracy & & Avg & Range \\
\hline EM-1 & $7 / 7$ & $7 / 7$ & $5 / 7$ & $3 / 7$ & $14 / 14$ & $11 / 14$ & $12 / 12$ & $8 \min 22 \mathrm{~s}$ & $4 \min 40 s-14 \min 55 s$ \\
\hline EM-2 & $6 / 6$ & $6 / 6$ & $5 / 6$ & $4 / 6$ & $11 / 12$ & $7 / 12$ & $10 / 10$ & $7 \min 35 s$ & $5 \min 35 \mathrm{~s}-10 \min 30 \mathrm{~s}$ \\
\hline EM-3 & $8 / 8$ & $7 / 8$ & $7 / 8$ & $6 / 8$ & $4 / 4$ & $4 / 4$ & $4 / 4$ & $6 \min 39 s$ & $4 \min 5 \mathrm{~s}-11 \min 30 \mathrm{~s}$ \\
\hline \multirow[t]{3}{*}{ Total } & $21 / 21$ & $20 / 21$ & $17 / 21$ & $13 / 21$ & $29 / 30$ & $22 / 30$ & $26 / 26$ & $7 \min 38 s$ & \\
\hline & & Sens 95 & & Sens 62 & & Sens 73 & Sens 100 & & \\
\hline & & CI $(74,99)$ & & CI $(39,81)$ & & CI $(54,87)$ & CI $(84,100)$ & & \\
\hline
\end{tabular}

$V H$ vitreous hemorrhage, $R H$ retro-bulbar hematoma, IOFB intraocular foreign body, $C R A$ central retinal artery, Abnl abnormal

orbital hematoma, intraocular foreign bodies, and central retinal artery flow. EM residents were able to detect significant abnormality greater than $93 \%$ of the time (see Table 1). Vitreous hemorrhage was the most detectable pathology with 95\% accuracy. One EM-3 falsely diagnosed the vitreous hemorrhage as a retinal detachment. A significant abnormality was detected in the models with intraocular foreign bodies $97 \%$ of the time with an accurate diagnosis of foreign body noted in $73 \%$ of the cases. One EM-2 inaccurately diagnosed the foreign body as normal, another diagnosed it as a vitreous hemorrhage, while three others called the scan abnormal without being able to posit a specific diagnosis. All EM-1s correctly identified the foreign bodies as abnormal but $3 / 14$ could not offer a specific diagnosis. Retro-orbital hemorrhages were the most difficult to diagnose with $62 \%$ accuracy. One EM-3 inaccurately diagnosed it as normal, while another diagnosed it as a retinal detachment. One EM-2 diagnosed it as normal while another called it abnormal without a specific diagnosis and two EM1s diagnosed the hematoma as normal, one misdiagnosed it as a foreign body, while one called it abnormal without a specific diagnosis. The average time taken for scanning two eyes was 7 min and $38 \mathrm{~s}$, with the EM-3s averaging 6 min and $39 \mathrm{~s}$ for scanning both eyes followed by the EM-2s at 7 min and $35 \mathrm{~s}$ and EM-1s at 8 min and $22 \mathrm{~s}$. Central retinal artery flow was detected in $100 \%$ of the 26 cases. Two residents did not have the opportunity to assess retinal artery flow because the pigs had expired, confirmed after thoracotomy. Ability to identify the abnormality ranged from 92\% among the EM-2s and 95\% among the EM-3s. Accuracy of diagnoses averaged $76 \%$ among all residents with the EM-2s accurately diagnosing pathology $65 \%$ of the time while the EM-3s were $85 \%$ accurate.

\section{Discussion}

Live tissue training is limited to those situations in which no adequate alternative is available. While many simulation models exist for ultrasound training, there are currently no ocular models. Additionally, no simulated model can reproduce real-time blood flow or tissue compliance as well as live tissue. Until a realistic ocular ultrasound model is produced, a live tissue model remains the best alternative. A universal challenge in training residents is obtaining proficiency in identifying diagnoses and performing procedures which do not occur frequently. Many of the EM-3s in the study population had not previously diagnosed a vitreous hemorrhage or retro-bulbar hematoma.

As with the more common emergency bedside ultrasound procedures of abdominal aortic aneurysm, focused assessment with sonography in trauma (FAST), first trimester ultrasound, and right upper quadrant ultrasound, we attempt to answer Yes-No questions with ocular ultrasound. The straightforward ocular anatomy combined with the simplicity of performing an ocular scan make learning ocular sonography beneficial.

\section{Limitations}

Our study contained several limitations. The main limitation of the study is the lack of gold standard by which to compare the residents' scans. All formal ocular scans conducted in our emergency department are followed up with a formal study such as CT or ophthalmological consultation for a more detailed examination. Although the porcine eye has been utilized in previous sonographic research, the model utilized has not been previously validated which introduced potential bias. The amount of blood introduced into the vitreous and retro-bulbar areas was selected by the researchers without knowledge of the average hematoma size in clinical practice. Additionally, the researchers fashioned metallic foreign bodies that were larger than those fashioned by Shiver, Lyon, and Blaivas [6]. The larger-sized hematoma and foreign body may introduce bias by increasing the chance that a novice 
sinologist will make a correct diagnosis. The equipment utilized was specifically for veterinary use and it was not feasible to utilize additional imaging modalities. The ultrasound machine utilized is equivalent to the Sonosite 180 , which is an early generation machine still in use. More advanced equipment with improved resolution and smaller, higher frequency transducers are available in many EDs and would lead to improved image clarity.

The resident sonographers were not given clinical information or allowed to visually inspect the eye. This ensured that they were relying solely on the ultrasound image for diagnosis. The porcine eye has been used extensively in human ophthalmological research and is generally considered a suitable model for the human eye [9-12]. Knowledge of preceding trauma or visual inspection identifying an irregularly shaped pupil or proptosis would be vital in the ED and would likely increase pretest probability of certain pathologies. The intent of the animal lab is to conduct a variety of procedures. This study was an adjunct and took place within the bounds of the procedure lab. For example, the animal was placed supine for optimal access for procedures. On a porcine model, unlike a human patient, the eyes are facing the floor while supine, making sonography more difficult than on a human subject whose eyes would face the ceiling while supine. Additionally, the sonographers remained at the head of the bed shielding them from other lab participants, whereas they may be able to stand nearer the torso of a human subject. In effect, the residents had to scan the pigs from an upside down and backwards position which increased their difficulty and thus their time to obtain adequate images.

Finally, in an ideal situation no pressure would be applied to the globe if globe rupture was a possibility. In our scenario, given multiple sonographers and the positioning of the animals, pressure was applied to the orbit which potentially affected the emplaced abnormalities. Hematomas could potentially disperse and other abnormalities such as a retinal detachment could be created from the repetitive scans.

\section{Conclusion}

After minimal training, EM residents are able to identify central retinal artery flow and ocular pathology in a porcine model with moderate accuracy. Central retinal artery flow and vitreous hemorrhage appear to be the easiest to diagnose with 100 and $95 \%$ accuracy, respectively. Intraocular foreign body and retro-bulbar hemorrhage were more difficult to diagnose with an accuracy of 73 and $62 \%$, respectively. With ocular ultrasound being in the early stages of acceptance in the emergency department, more research in this area is warranted.

Conflict of interest None.

\section{References}

1. McGwin G Jr, Xie A, Owsley C (2005) Rate of eye injury in the United States. Arch Ophthalmol 123(7):970-976

2. American College of Emergency Physicians (2008) ACEP Policy Statement: emergency ultrasound guidelines. pp 1-38. American College of Emergency Physicians Web site at https://www.acep. org/WorkArea/DownloadAsset.aspx?id=32878

3. Blaivas M (2000) Bedside emergency ultrasound in the evaluation of ocular pathology. Acad Emerg Med 7:947-950

4. Williams SR, Ahuja Y (2005) Ultrasonographic diagnosis of retinal detachment in the emergency department. Ann Emerg Med. 45:98 (Letter to Editor)

5. Lyon M, Blaivas M, Brannam L et al (2005) Use of emergency ultrasound in a rural ED with limited radiology services. Am J of Emerg Med 23:212-214 (Letter to Editor)

6. Shiver SA, Lyon M, Blaivas M (2005) Detection of metallic ocular foreign bodies with handheld sonography in a porcine model. J Ultrasound Med 24:1341-1346

7. Legome E, Pancu D (2004) Future applications for emergency ultrasound. Emerg Med Clin N Am 22:817-827

8. Tayal VS et al (2007) Emergency department sonographic measurement of optic nerve sheath diameter to detect finding of increased intracranial pressure in adult head injury patients. Ann Emerg Med 49:508-514

9. Ruiz-Ederra $J$ et al (2005) The pig eye as a novel model of glaucoma. Exp Eye Res 81:561-569

10. Reichl S, Bednarz J, Muller-Goyman CC (2004) Human corneal equivalent as cell culture model for in vitro drug permeation studies. Br J Ophthalmol 88:560-565

11. Olsen TW et al (1998) Human sclera: thickness and surface area. Am J Ophthalmol 125:237-241

12. Olsen TW et al (2002) Porcine sclera: thickness and surface area. Invest Ophthalmol Vis Sci 43:2529-2532 